\title{
EMIM离子液体离子簇模型的量子化学计算
}

\author{
李 巍1, 张 静 $^{2}$ 戚传松 \\ ('北京石油化工学院化学工程学院, 北京 102617; ${ }^{2}$ 中国科学院大学材料科学与光电技术学院, 北京 100049)
}

\begin{abstract}
摘要: 以1-乙基-3-甲基咪唑(EMIM)卤化物、氟硼酸盐、三澳化物和二碘溴酸盐、氯铝酸和溴铝酸盐等不同 种类EMIM离子液体为研究对象, 对多阳离子、多阴离子的离子簇模型进行了量子化学计算研究. 首先在 B3LYP/6-311++G $(d, p)$ 水平上(I使用6-311G $(d, p)$ 基组)对 $\left\{[E M I M] X_{n}\right\}^{(n-1)-}\left(\mathrm{X}=\mathrm{Cl}, \mathrm{Br}, \mathrm{I}, \mathrm{BF}_{4}, \mathrm{AlCl}_{4}, \mathrm{AlBr}_{4}, \mathrm{Br}_{3}\right.$, $\mathrm{IBrl}, \mathrm{FHF} ; n=2,3)$ 和 $\left\{[\mathrm{EMIM}]_{2} \mathrm{X}_{n}\right\}^{\left(n^{\prime}-2\right)-}\left(n^{\prime}=3,4,5\right)$ 离子簇进行构型优化, 并对卤化物和氟嗍酸盐进行了振动 光谱计算. 结果表明所采用理论模型在键长、键角等结构参数及红外振动光谱方面均与实验结果符合较好. 同时对不同离子簇模型中阴、阳离子间相互作用能与实验熔点之间的关系进行了研究, 发现采用 $\left\{[E M I M]_{2} X_{n}\right\}^{\left(n^{2}-2\right)}$ 模型时EMIM离子液体实验熔点与阴、阳离子间相互作用能之间呈现近线性关系.
\end{abstract}

关键词：密度泛函理论；咪唑离子液体；相互作用能；离子簇；熔点

中图分类号: 0641

\section{Quantum Chemistry Calculations of Ion Cluster Models of EMIM Ionic Liquids}

\author{
LI Wei $^{1, *} \quad$ ZHANG Jing ${ }^{2} \quad$ QI Chuan-Song ${ }^{1}$ \\ ( ${ }^{1}$ College of Chemical Engineering, Beijing Institute of Petro-Chemical Technology, Beijing 102617, P. R. China; \\ ${ }^{2}$ College of Materials Science and Optoelectronics Technology, University of Chinese Academy of Sciences, Beijing 100049, P. R. China)
}

\begin{abstract}
Different types of 1-ethyl-3-methylimidazolium (EMIM) ionic liquid compounds, including halides, tetrafluoroborate, tribromide, diiodobromate, chloroaluminate, and bromine aluminate, have been investigated using quantum chemical calculations. First, geometry optimizations of the ion systems, including $\left\{[\mathrm{EMIM}] \mathrm{X}_{n}\right\}^{(n-1)-}\left(\mathrm{X}=\mathrm{Cl}, \mathrm{Br}, \mathrm{I}, \mathrm{BF}_{4}, \mathrm{AlCl}_{4}, \mathrm{AlBr}_{4}, \mathrm{Br}_{3}, \mathrm{IBrl}, \mathrm{FHF} ; n=2,3\right)$ and $\left\{[\mathrm{EMIM}]_{2} \mathrm{X}_{n}\right\}^{\left(n^{\prime}-2\right)-}\left(n^{\prime}=3,4,5\right)$, were performed using the density functional theory (DFT) B3LYP method together with the 6-311++G $(d, p)(6-$ $311 \mathrm{G}(d, p)$ for I) basis set. The vibrational spectra were also calculated for the EMIM halides and tetrafluoroborate. The obtained structures and vibrational spectra were consistent with experimental results. In addition, a linear correlation between melting point and interaction energy was obtained for the $\left\{[E M I M]_{2} \mathrm{X}_{n^{\prime}}\right\}^{\left(n^{\prime}-2\right)-}$ models of the compounds studied.
\end{abstract}

Key Words: Density functional theory; Imidazolium ionic liquid; Interaction energy; Ion cluster; Melting point

1 引言

室温离子液体(RTILs, 简称ILs) 是由不对称的
有机阳离子与无机或有机阴离子构成的一类新型 溶剂, 由于其环保性好等特点被认为是传统有机溶

Received: March 30, 2015; Revised: July 6, 2015; Published on Web: July 7, 2015.

*Corresponding author. Email: liwei77@bipt.edu.cn; Tel: +86-10-81292127.

The project was supported by the Breeding Project of Outstanding Academic Leaders of Beijing Institute of Petro-Chemical Technology, China (BIPT-BPOAL-2014).

北京石油化工学院优秀学科带头人培育计划(BIPT-BPOAL-2014)资助项目

(C) Editorial office of Acta Physico-Chimica Sinica 
剂的有力替代者; ${ }^{1-4}$ 而增加离子液体的种类、充实 离子液体化学的基础内容、开辟离子液体新的研 究与应用领域是绿色化学的需要. 有机阳离子与阴 离子之间可能的组合数目庞大, 但是能够满足特定 应用需求的组合方式是有限的, 因而离子液体经常 被称为“designer solvents”, 而如何设计出具有特定 性质及功能的离子液体是当前的一个研究热点. 目 前离子液体的设计仍然是以一种比较盲目的“tryand-error” 模式进行, 并没有真正做到根据目标进行 设计. 为了能够从分子水平上设计低熔点、功能化 的离子液体, 以及在新型离子液体的探索中避免人 力及物力的浪费, 离子液体基础性质及功能预测的 理论研究势在必行.

而离子液体的理论计算研究相对于其实验研 究发展较慢, 这主要是由于对于凝聚相体系至今还 没有发展出一个高精度和高效性两者兼备的算法. 目前常用的理论手段包括使用量子化学计算方法 研究气相中离子对结构, 或是构建力场、利用分子 模拟方法研究离子液体的宏观物理性质, 以及利用 统计方法结合量子化学 ${ }^{5-14}$ 或半经验方法 ${ }^{15-19}$ 进行定 量构效关系(QSPR)研究. 在过去的 10 年里, 基于分 子描述符的QSPR研究发展迅速, 在相关的研究中 多数采用半经验方法或分子力学方法优化构型, 参 数的选择主要服务于精度的需要, 对预测结果影响 较大的参数数量较多, 对物理化学性质的影响因素 只能定性的分析; 同时, 方法应用的效果依赖于算 法及个人经验, 至今为止还没有一种普适于不同种 类离子液体的构效关系表达式.

在构效关系研究方面我们的目标是力图发现 一种普适性强、形式简单、物理意义明确的构效 关系式, 而目前我们最关注的实验性质是熔点, 这 是由于低熔点是离子液体具有应用前景的首要条 件; 此外 $\mathrm{He}$ 等 ${ }^{20}$ 发现对于 $N$-取代的甘氨酸酯化物阳 离子型离子液体, 熔点与黏度呈现正相关性; Rahman等 ${ }^{21}$ 在研究四乙铵氨基酸离子液体时则发现 其离子导电性与黏度成线性相关关系. 可见, 熔点 是离子液体非常重要的一项性质指标. 考虑到离子 液体是一种以库仑相互作用为主的体系, 而在发生 熔化过程体积发生膨胀时需要克服的阻力以阴阳 离子间相互作用为主, 因此我们以量子化学计算获 得的离子间相互作用为唯一的描述符, 试图建立离 子液体的熔点与离子间相互作用的定量关系. 我们 已经在氨基酸阳离子型离子液体、 ${ }^{5}$ 烷基咪唑氟嗍
酸离子液体 ${ }^{6}$ 和烷基咪唑卤化物 7 中进行了尝试并获 得一定的成功. 类似的尝试也表现在部分其他小组 的工作中, 但多数以失败告终, 其中 $\mathrm{Wu}^{8}$ 和Mohajeri ${ }^{9}$ 等均对咪唑氨基酸离子液体的离子对进行了系统 地量子化学计算研究; 而Turner ${ }^{10}$ 及Katsyuba小组 ${ }^{1}$ 分 别系统研究了咪唑卤化物及咪唑氟嗍酸系列离子 液体的离子对结构. 上述工作在咪唑离子液体研究 时未能获得与实验熔点与某种分子结构性质的相 关性的主要原因是由于他们均采用单个阳离子与 单个阴离子组成的离子对模型, 而此模型不能真正 反映凝聚相体系中真实的结构特征. 6,7 我们之前在 研究咪唑类化合物时选择一个咪唑阳离子与 $2-3$ 个 阴离子组成的一价或二价分子离子为研究对象, 发 现量子化学计算优化获得的结构与晶体结构实验 结果更加符合, 且阴、阳离子间相互作用能与实验 熔点之间存在线性关系. 6.7 在之前关于咪唑类离子 液体的构效关系研究中, 基本是以单一种类阴离子 的体系为研究对象. 在本文中, 我们在前期工作的 基础上扩展研究对象, 对阴离子种类不同的常见1乙基-3-甲基咪唑(EMIM)离子液体进行量子化学计 算研究, 采用多个阳离子和多个阴离子组成的离子 团簇模型, 考虑不同阴离子与EMIM阳离子间的作 用模式, 并进一步研究熔点与离子间相互作用能之 间的构效关系.

\section{2 研究体系及研究方法}

\section{1 研究体系}

我们之前研究了阴离子相同的咪唑类离子液 体实验熔点与离子间相互作用能之间的关系, 在本 次工作中我们选择阳离子均为 1 -乙基-3-甲基咪唑 的离子液体为研究对象, 通过检索Reaxys数据库 ${ }^{22}$ 收 集具备实验熔点的1-乙基-3-甲基咪唑化合物; 同时 结合检索剑桥晶体数据库, 选择既具有实验熔点又 具备晶体结构信息的常见1-乙基-3-甲基咪唑化合物 作为研究对象, 具体包括: 氟嗍酸盐([EMIM] $\left[\mathrm{BF}_{4}\right]$ )、氟 化物([EMIM][FHF])、氯化物([EMIM]Cl)、溴化物 $([\mathrm{EMIM}] \mathrm{Br})$ 、碘化物 ([EMIM]I)、三溴化物 $\left([\mathrm{EMIM}]\left[\mathrm{Br}_{3}\right]\right)$ 及澳铝酸盐 $\left([\mathrm{EMIM}]\left[\mathrm{AlBr}_{4}\right]\right)$. 此外, 氯 铝酸盐([EMIM] $\left.\left[\mathrm{AlCl}_{4}\right]\right)$ 、二碘澳酸盐([EMIM] [IBrI]) 虽然未查到晶体结构信息, 但由于其阴离子部分与 已知晶体结构的化合物具有相似性, 在本文工作中 也进行了讨论.

\section{2 理论模型的选择}


通过检索剑桥晶体数据库, 得到了百余种 $1-乙$ 基-3-甲基咪唑化合物的晶体结构信息. 通过观察、 对比发现阴离子的尺寸、对称性对晶体中离子的 排列有着至关重要的影响, 从而进一步影响离子间 的相互作用模式. 本文讨论的常见EMIM离子液体 中阴离子从小至大排列为(括号内数值为估算得到 的离子外接球半径, 其中多原子阴离子按照晶体结 构中键长数据及端原子范德华半径 ${ }^{23}$ 估算得到): $\mathrm{Cl}^{-}$

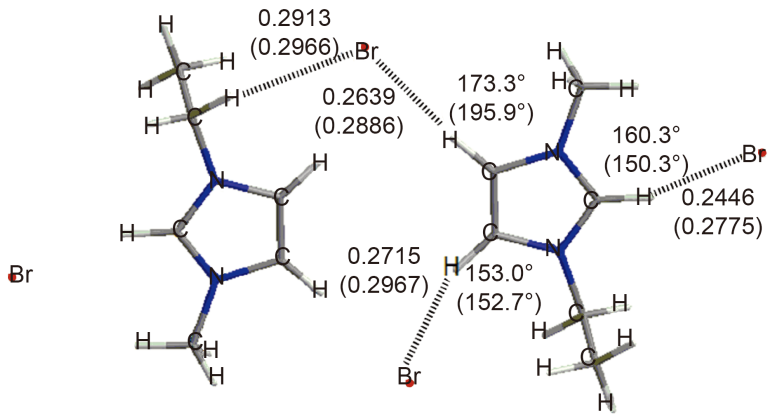

(a)

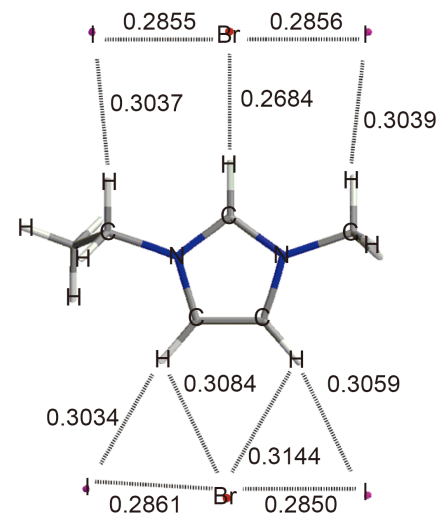

(c)

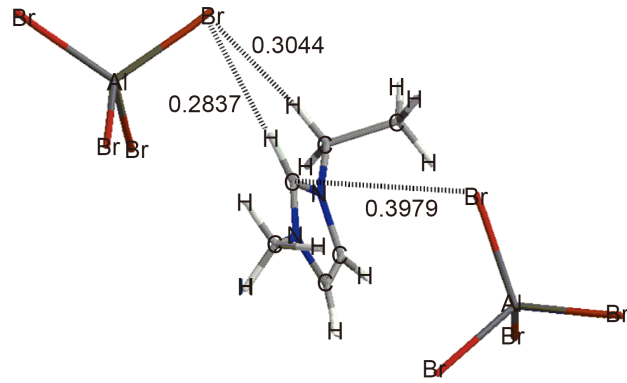

(e)
$(0.18 \mathrm{~nm}) 、 \operatorname{Br}^{-}(0.20 \mathrm{~nm}) 、 \mathrm{I}^{-}(0.22 \mathrm{~nm}) 、 \mathrm{FHF}^{-}(0.25$ $\mathrm{nm}) 、 \mathrm{BF}_{4}^{-}(0.28 \mathrm{~nm}) 、 \mathrm{AIBr}_{4}^{-}(0.32 \mathrm{~nm}) 、 \mathrm{Br}_{3}^{-}(0.44$ $\mathrm{nm})$. 阴离子尺寸及对称性相近时晶体结构中阴、 阳离子的排布模式也相似. 我们选择能够反映晶体 结构中阴、阳离子相互作用模式的最小结构单元 作为理论模型, 具体选择的离子簇如图 1 和图 2 所示, 具体分析详见结果与讨论部分.

在我们之前的工作 ${ }^{6,7}$ 中曾经对烷基咪唑卤化物

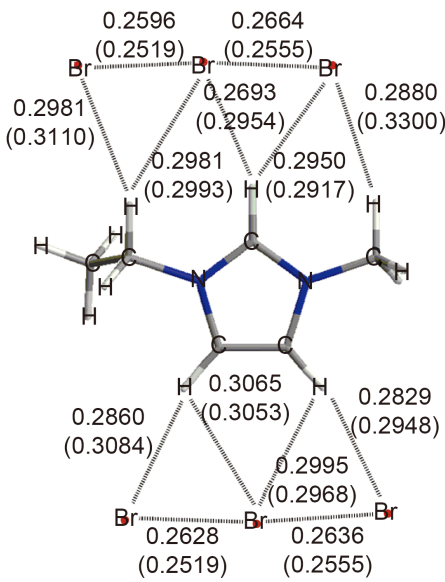

(b)

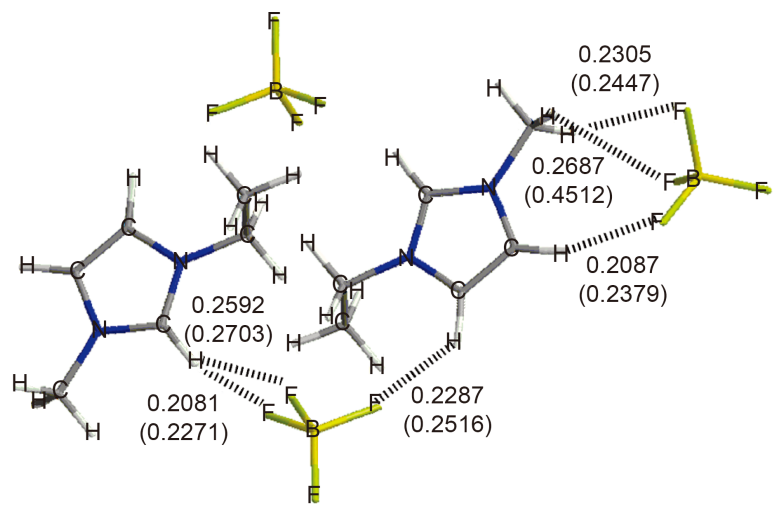

(d)

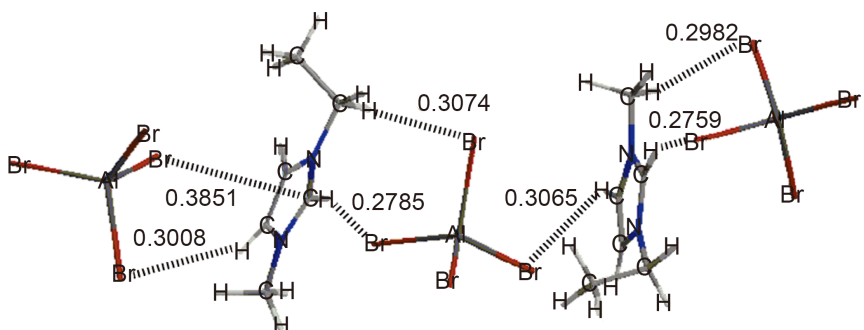

图1 EMIM离子液体离子簇优化构型

Fig.1 Optimized structures of ion clusters of EMIM ILs

(a) $\left\{[\mathrm{EMIM}]_{2} \mathrm{Br}_{4}\right\}^{2-}$; (b) $\left\{[\mathrm{EMIM}]\left[\mathrm{Br}_{3}\right]_{2}\right\}^{-}$; (c) $\left\{[\mathrm{EMIM}][\mathrm{IBrI}]_{2}\right\}^{-}$; (d) $\left\{[\mathrm{EMIM}]_{2}\left[\mathrm{BF}_{4}\right]_{4}\right\}^{2-}$; (e) $\left\{[\mathrm{EMIM}]\left[\mathrm{AlBr}_{4}\right]_{2}\right\}^{-}$; (f) $\left\{[\mathrm{EMIM}]_{2}\left[\mathrm{AlBr}_{4}\right]_{3}\right\}^{-}$. bond length in $\mathrm{nm}$. The data in the parentheses are experimental values from Cambridge Structural Database. 


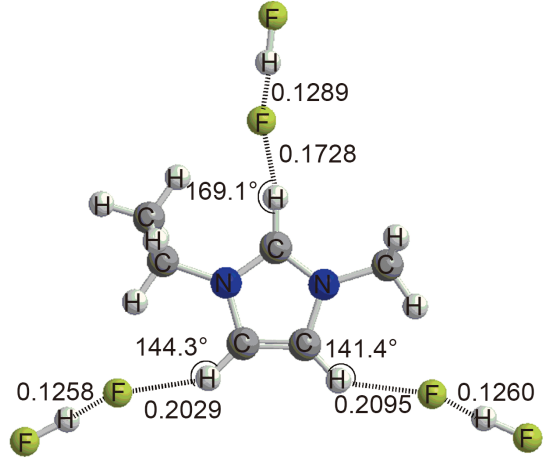

(a)

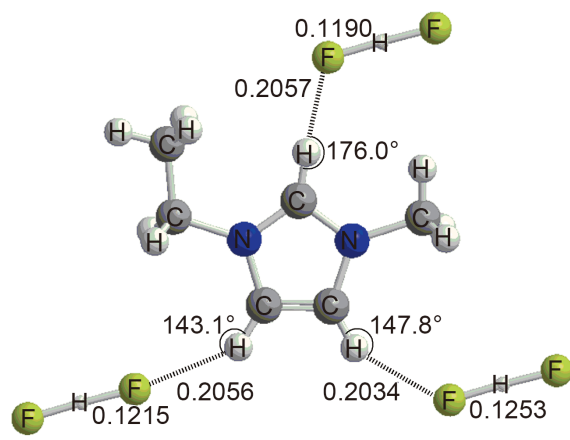

(b)

图2 $\left\{\right.$ [EMIM] $\left[\mathrm{FHF}_{3}\right\}^{2-}$ 优化构型及晶体结构中相应结构单元

Fig.2 Optimized geometry of $\left\{[\mathrm{EMIM}][\mathrm{FHF}]_{3}\right\}^{2-}$ and corresponding geometry in crystal

(a) theoretically optimized geometry; (b) structural unit in crystal. bond length in $\mathrm{nm}$

及烷基咪唑氟嗍酸盐进行过系统的量子化学计算 研究, 当时采用的模型是 $\left\{[\mathrm{EMIM}] \mathrm{X}_{n}\right\}^{(n-1)-}(n=2,3)$, 这一模型比较好地反映了直接与咪唑阳离子作用 的周围阴离子的排布情况, 并且从该模型出发得到 了离子间相互作用能与实验熔点之间的线性关系. 但该模型仍然存在不足: 首先, 它并不适用于较大 阴离子体系, 如 $[\mathrm{EMIM}]\left[\mathrm{Br}_{3}\right] 、[\mathrm{EMIM}]\left[\mathrm{AlBr}_{4}\right]$ 等情 况; 此外, 对于氟硼酸体系该模型中 B原子与咪唑环 几乎在同一平面内, 与实际晶体结构不完全一致. 在本文中我们将选取能够更好反映实际晶体结构 特征的离子簇模型作为研究对象, 探究阴离子种类 不同的咪唑离子液体中离子间的作用模式.

\section{3 量子化学计算}

基于Gaussian 03软件包, ${ }^{24}$ 采用密度泛函理论 (DFT)方法B3LYP和 6-311++G $(d, p)$ 基组(I使用6$311 \mathrm{G}(d, p)$ 基组), 对不同离子簇进行了构型优化并 对部分离子簇优化构型进行了振动频率分析, 将得 到的理论振动光谱与实验结果进行对比, 帮助指认 实验红外光谱谱线的同时对不同理论模型的结果 进行了对比. 此外, 利用超分子方法计算了离子簇 中离子间相互作用能, 即 $\Delta E=E^{\mathrm{A}_{n} \mathrm{~B}_{m}}-n E^{\mathrm{A}}-$ $m E^{\mathrm{B}}\left(E^{\mathrm{A}}, E^{\mathrm{B}}\right.$ 及 $E^{\mathrm{A}_{n} \mathrm{~B}_{m}}$ 分别代表经过结构优化的阳离 子、阴离子及离子簇能量), ${ }^{25}$ 并且采用Counterpoise 方法进行了基组重叠效应(BSSE)修正, ${ }^{26-28}$ 进而讨论 离子间相互作用能与实验熔点之间的关系.

\section{3 结果与讨论}

\section{1 优化构型及振动光谱}

各离子簇的优化构型见图1及图2. 其中部分重 要结构参数列于图中(图中括号部分为实验晶体结
构中对应的数值, 键长量纲为 $\mathrm{nm}$ )及相应表格中. 下 面将对不同的体系进行具体分析.

\subsection{1 卤化物}

$\left\{[\mathrm{EMIM}]_{2} \mathrm{Br}_{4}\right\}^{2-}$ 的优化构型参见图1(a), 碘化物 与氯化物的优化构型和溴化物相似, 未给出具体构 型图, 相应结构参数列于表1中(优化结果具有对称 性, 仅列出一组数据). 如图1(a)所示的结构单元采用 两种不同的取向在空间交错排布构成周期性结构.

溴化物与碘化物的优化结果均与晶体结构中 的相应结构参数符合较好, 从图1(a)及表1列出的具 体数值分析, $\mathrm{Br} \cdots \mathrm{H}$ 距离理论结果比实验值小 $0.025-0.033 \mathrm{~nm}, \mathrm{Br} \cdots \mathrm{C}$ 距离均比实验值小 $0.010 \mathrm{~nm}$; $\mathrm{I} \cdots \mathrm{C}$ 的距离与实验值比较小 $0.005 \mathrm{~nm}$ 左右, 这主要 是由于未考虑周围离子环境而孤立考虑离子簇所 造成的. 之前氯化物晶体结构的报道中 $\mathrm{C} \cdot \mathrm{C}$ 的平 均键长为 $0.355 \mathrm{~nm}$, ${ }^{29}$ 优化结构中 $\mathrm{C} \cdots \mathrm{Cl}$ 的平均值为 $0.346 \mathrm{~nm}$, 与实验值符合较好.

为了进行对比, $\left\{[\mathrm{EMIM}] \mathrm{X}_{3}\right\}^{2-}(\mathrm{X}=\mathrm{Br}, \mathrm{I})$ 模型的 优化构型参数 ${ }^{7}$ 也列于表 1 中. 可以发现 $\left\{[\mathrm{EMIM}]_{2} \mathrm{X}_{4}\right\}^{2-}$ 和 $\left\{[\mathrm{EMIM}] \mathrm{X}_{3}\right\}^{2-}$ 模型中的结构参数都比较好地接近 实验晶体结构中的数据, 而 $\left\{[\mathrm{EMIM}]_{2} \mathrm{X}_{4}\right\}^{2-}$ 模型在键 角描述方面更接近实验值, 且从描述离子间的相互 作用模式来说更全面, 同时从后面3.2节的讨论中还 可以看到采用 $\left\{[\mathrm{EMIM}]_{2} \mathrm{X}_{4}\right\}^{2-}$ 模型后其离子间相互 作用能与实验熔点之间的定量关系与其他种类阴 离子的EMIM化合物符合更好.

卤化物振动光谱的实验数据及不同离子簇模 型的理论计算值列于表 2 中, 其中对于实验光谱峰 的指认主要是根据与 $\left\{[\mathrm{EMIM}]_{2} \mathrm{X}_{4}\right\}^{2-}$ 模型理论值的 比较分析来实现. 各离子簇模型的计算结果均无虚 
表1 EMIM卤化物晶体结构及各优化构型结构参数

Table 1 Structural parameters in crystal and optimized ion clusters of EMIM halides

\begin{tabular}{|c|c|c|c|c|c|c|}
\hline & $A(\mathrm{X}-\mathrm{H}-\mathrm{C} 2) /\left(^{\circ}\right)$ & $A(\mathrm{X}-\mathrm{H}-\mathrm{C} 4) /\left({ }^{\circ}\right)$ & $A(\mathrm{X}-\mathrm{H}-\mathrm{C} 5) /\left(^{\circ}\right)$ & $L(\mathrm{X}-\mathrm{C} 2) / \mathrm{nm}$ & $L(\mathrm{X}-\mathrm{C} 4) / \mathrm{nm}$ & $L(\mathrm{X}-\mathrm{C} 5) / \mathrm{nm}$ \\
\hline$[\mathrm{EMIM}] \mathrm{Br}^{\mathrm{a}}$ & 150.3 & 195.9 & 152.7 & 0.3575 & 0.3820 & 0.3817 \\
\hline$\left\{[\mathrm{EMIM}]_{2} \mathrm{Br}_{4}\right\}^{2-}$ & 160.3 & 173.3 & 153.0 & 0.3495 & 0.3720 & 0.3714 \\
\hline$\left\{[\mathrm{EMIM}] \mathrm{Br}_{3}\right\}^{2-}$ & 175.8 & 146.2 & 145.8 & 0.3557 & 0.3875 & 0.3896 \\
\hline 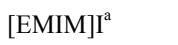 & 144.0 & 195.4 & 155.8 & 0.3819 & 0.4048 & 0.4028 \\
\hline$\left\{[\mathrm{EMIM}]_{2} \mathrm{I}_{4}\right\}^{2-}$ & 160.5 & 167.9 & 153.0 & 0.3770 & 0.3991 & 0.4006 \\
\hline$\left\{[\mathrm{EMIM}] \mathrm{I}_{3}\right\}^{2-}$ & 175.9 & 149.7 & 150.2 & 0.3856 & 0.4097 & 0.4074 \\
\hline$\left\{[\mathrm{EMIM}]_{2} \mathrm{Cl}_{4}\right\}^{2-}$ & 158.3 & 178.1 & 152.0 & 0.3314 & 0.3532 & 0.3524 \\
\hline
\end{tabular}

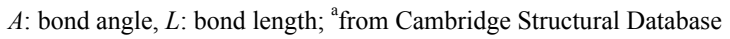

频. 由于 $\left\{[\mathrm{EMIM}]_{2} \mathrm{X}_{4}\right\}^{2-}$ 模型相对于其在晶体中的结 构更加紧凑, 可以看到咪唑环 $\mathrm{C}-\mathrm{H}$ 的伸缩振动频率 虽然接近实验值但略高于实验值. 表2中涉及甲基 和乙基的振动模式的理论计算结果明显偏离实验 结果, 这主要是因为所采用模型并未考虑甲基和乙 基周围环境的影响. 我们根据Scott和Radom ${ }^{30}$ 的处理 方法, 利用 $\left\{[\mathrm{EMIM}]_{2} \mathrm{X}_{4}\right\}^{2-}(\mathrm{X}=\mathrm{Cl}, \mathrm{Br}, \mathrm{I})$ 模型的甲基 和乙基振动模式的计算结果, 得到校正因子 0.9384 . 表 2 中括号数值即为原有计算结果乘以校正因子后 的数值, 经校正后与实验结果符合较好. 而其他振 动模式, 如咪唑环上的 $\mathrm{C}-\mathrm{H}$ 伸缩振动等, 是在考虑 了周围离子环境条件下计算得到的, 因此未作进一 步校正. [EMIM]Cl离子对的最稳构型及 $\left\{[\mathrm{EMIM}] \mathrm{Cl}_{3}\right\}^{2-}$ 模型的振动光谱计算结果也列于表 2 中, 其振动模 式与 $\left\{[\mathrm{EMIM}]_{2} \mathrm{X}_{4}\right\}^{2-}$ 模型不同的直接列于相应数值 下方. 通过对比不难发现各模型在咪唑环甲基和乙 基的振动情况描述方面差别不大；但在咪唑环
$\mathrm{C}-\mathrm{H}$ 的伸缩振动描述上本研究中所采用的模型与 实验值 ${ }^{31}$ 符合更好，而离子对模型的结果明显偏离 实验值.

\section{1 .2 氟硼酸盐}

与卤化物情况相似, 在EMIM氟嗍酸盐晶体结 构中, 如图1(d)所示的结构单元采用两种不同取向 在空间交错排布形成周期性结构. $\left\{[\mathrm{EMIM}]_{2}\left[\mathrm{BF}_{4}\right]_{4}\right\}^{2-}$ 优化构型与实验晶体结构中的相应单元比较, 咪唑 环的排布相对松散: 晶体结构中咪唑环中心连线与 咪唑环夹角大于 $\left\{[\mathrm{EMIM}]_{2}\left[\mathrm{BF}_{4}\right]_{4}\right\}^{2-}$ 优化构型中的相 应夹角(均取锐角); 咪唑环之间垂直距离实验值比 理论模型值短约 $0.01 \mathrm{~nm}$. 在我们之前工作中采用的 $\left\{[\mathrm{EMIM}]\left[\mathrm{BF}_{4}\right]_{3}\right\}^{2-}$ 模型中阴离子的中心 $\mathrm{B}$ 原子与咪唑 环几乎在同一平面, 而 $\left\{[\mathrm{EMIM}]_{2}\left[\mathrm{BF}_{4}\right]_{4}\right\}^{2-\text { 优化构型中 }}$ 与咪唑环 $\mathrm{C} 2$ 原子相近的 $\mathrm{BF}_{4}^{-}$阴离子中心 $\mathrm{B}$ 原子与 EMIM咪唑环不同面, 后者更加符合晶体结构中的 离子排布特征.

表2 [EMIM]X红外光谱实验及理论计算值 $\left(\right.$ 单位 $\left.\mathbf{c m}^{-1}\right)$

Table 2 Experimental and calculated IR spectrum data (unit in $\mathrm{cm}^{-1}$ ) of [EMIM]X

\begin{tabular}{|c|c|c|c|c|c|c|c|c|}
\hline \multicolumn{3}{|c|}{ Experimental IR spectra $^{31}$} & \multirow{2}{*}[\mathrm{EMIM}]{$\mathrm{Cl}^{7}$} & $\multirow{2}{*}{[\mathrm{EMIM}] \mathrm{Cl}_{3}}^{2-7}$ & $\multirow{2}{*}{[\mathrm{EMIM}]_{2} \mathrm{Cl}_{4}}^{2}$ & $\multirow{2}{*}{[\mathrm{EMIM}]_{2} \mathrm{Br}_{4}}^{2}$ & $\multirow{2}{*}{[\mathrm{EMIM}]_{2} \mathrm{I}_{4}}^{2-}$ & \multirow{2}{*}{ Assignment } \\
\hline $\mathrm{Cl}$ & $\mathrm{Br}$ & I & & & & & & \\
\hline 3135 & 3137 & 3133 & 3271 & 3182 & 3109,3103 & 3139,3134 & 3173,3169 & $v \mathrm{C} 4-\mathrm{H}$ \\
\hline \multirow[t]{2}{*}{3154} & 3064 & 3080 & $\begin{array}{c}(v \mathrm{C} 4-\mathrm{H}, \mathrm{C} 5-\mathrm{H}) \\
3290\end{array}$ & $\begin{array}{c}(v \mathrm{C} 4-\mathrm{H}, \mathrm{C} 5-\mathrm{H}) \\
3201\end{array}$ & 3179 & 3187 & 3211 & $v \mathrm{C} 5-\mathrm{H}$ \\
\hline & & 3052 & $(v \mathrm{C} 4-\mathrm{H}, \mathrm{C} 5-\mathrm{H})$ & $(v \mathrm{C} 4-\mathrm{H}, \mathrm{C} 5-\mathrm{H})$ & & & & \\
\hline 2962 & 2959 & 2963 & 2610 & 2971 & 2988 & 3020 & 3074 & $v \mathrm{C} 2-\mathrm{H}$ \\
\hline \multirow[t]{5}{*}{2927} & 2925 & 2926 & 3132 & 3124 & $3127(2934)$ & $3130(2937)$ & $3137(2944)$ & $v_{\text {as }} \mathrm{Me}$ \\
\hline & & & 3117 & 3100 & & & & \\
\hline & & & 3119 & 3104 & $3120(2928)$ & $3122(2930)$ & $3121(2929)$ & $v_{\text {as }} \mathrm{Et}$ \\
\hline & & & 3109 & 3090 & 3094(2903) & $3103(2912)$ & $3104(2913)$ & \\
\hline & & & 3096 & 3080 & & $3097(2906)$ & $3099(2908)$ & \\
\hline 2856 & 2858 & 2854 & 3063 & $\begin{array}{c}3023,3016 \\
\left(v_{\mathrm{s}} \mathrm{Et}\right)\end{array}$ & $3061(2872)$ & $3055(2867)$ & $3055(2867)$ & $v_{\mathrm{s}} \mathrm{CH}_{2}(\mathrm{Et})$ \\
\hline \multirow[t]{2}{*}{2850} & 2850 & 2850 & 3030 & 3001 & $3039(2852)$ & $3044(2856)$ & $3048(2860)$ & $v_{\mathrm{s}} \mathrm{CH}_{3}(\mathrm{Me})$ \\
\hline & & & 3033 & & $3029(2842)$ & $3032(2845)$ & $3033(2846)$ & $v_{\mathrm{s}} \mathrm{CH}_{3}(\mathrm{Et})$ \\
\hline
\end{tabular}


对EMIM氟硼酸盐的簇模型也进行了振动频率 计算, 虽然 $\left\{[\mathrm{EMIM}]_{2}\left[\mathrm{BF}_{4}\right]_{4}\right\}^{2-}$ 的计算结果中存在虚 频, 但考虑到该模型的初始构型是从EMIM氟硼酸 晶体结构中的相应单元出发, 因此没有考虑其他可 能的构型. EMIM氟硼酸盐的红外光谱实验值 ${ }^{11}$ 及不 同理论模型计算值列于表 3 中. Katsyuba等 ${ }^{11}$ 对 $[\mathrm{EMIM}]\left[\mathrm{BF}_{4}\right]$ 离子对模型进行了细致的研究, 但无 论哪种离子对的稳定构型其红外光谱中缺失对应 于实验值 $1619 \mathrm{~cm}^{-1}$ 的振动模式, 在我们的离子簇模 型中指认该振动模式为咪唑环 $\mathrm{C} 2-\mathrm{N} 3$ 与 $\mathrm{C} 4-\mathrm{C} 5$ 的 反对称协同振动; 此外, 该文献中 $1576 \mathrm{~cm}^{-1}$ 被指认 为 $\mathrm{C}=\mathrm{C}$ 振动，而我们的计算结果中其对应于 $\mathrm{N} 1-\mathrm{C} 2-\mathrm{N} 3$ 反对称伸缩. 由于所研究离子簇模型未 考虑周围其他离子的影响, 得到的阴、阳离子间距 离小于实验值, 咪唑环 $\mathrm{C}-\mathrm{H}$ 振动频率均高于实验 值. 咪唑甲基和乙基的相应振动频率采用卤化物中 得到的修正因子 0.9384 进行修正(表3中括号中数值), 修正后结果与实验值符合较好.

\section{1 .3 氟化物}

对于线性阴离子，如 $\mathrm{FHF}^{-}$和 $\mathrm{Br}_{3}^{-}$离子，其与 EMIM形成的化合物晶体结构呈层状排布; 但由于 二者尺寸相差较大, 每层中离子的排布方式并不相 同. 在 $[\mathrm{EMIM}][\mathrm{FHF}]$ 的晶体结构中, 每一层中每个阳 离子直接与 3 个阴离子相互作用, 而每个阴离子直 接与 3 个阳离子直接发生相互作用. $\left\{[\mathrm{EMIM}][\mathrm{FHF}]_{3}\right\}^{2}$ 的优化构型和晶体结构中的重复单元结构如图2所 示. 从图中可以看出由于空间排布的需要, 位于 $\mathrm{C} 2$ 及 $\mathrm{C} 4$ 附近的阴离子取向相对于气相中 $\left\{[\mathrm{EMIM}][\mathrm{FHF}]_{3}\right\}^{2-}$
单独存在时发生改变.

\section{1 .4 三溴化物及二碘溴酸盐}

$[\mathrm{EMIM}]\left[\mathrm{Br}_{3}\right]$ 晶体结构中沿 $c$ 轴方向咪唑阳离子 与 $\mathrm{Br}_{3}^{-}$交错呈列状排布, 根据这一特点选择如图 1(b)所示的 $\left\{[\mathrm{EMIM}]\left[\mathrm{Br}_{3}\right]_{2}\right\}$ 离子簇模型并进行优化. 由图中数据可见优化构型与相应晶体结构中对应 部分的差别主要在于离子簇中咪唑环上方 $\mathrm{Br}_{3}^{-}$略偏 向乙基一侧，下方 $\mathrm{Br}_{3}^{-}$略偏离直线结构(键角为 $\left.173.8^{\circ}\right)$, 同时 $\mathrm{Br}_{3}{ }^{-}$在所研究离子簇中更加松散. 类似 地对 $\left\{[\mathrm{EMIM}][\mathrm{IBrI}]_{2}\right\}$-进行结构优化, 结果列于图 $1(\mathrm{c})$. 同时为了建立离子间相互作用能与实验熔点 之间的定量关系, 对呈列状排布的 $\left\{[\mathrm{EMIM}]_{2}\left[\mathrm{Br}_{3}\right]_{3}\right\}$ 和 $\left\{[\mathrm{EMIM}]_{2}[\mathrm{IBrI}]_{3}\right\}$ 模型也进行了优化和能量计算.

\section{1 .5 氯铝酸盐及溴铝酸盐}

对于EMIM溴铝酸盐, 阴、阳离子尺寸相当, 晶 体结构中离子的空间排布更加规律，阴、阳离子沿 $a$ 轴及 $c$ 轴均呈列状排布, 且阴、阳离子列呈锯齿状 交替排布(参见图1(f)). 本文中研究的离子簇模型描 述阴、阳离子交替排布的情况.

$\left\{[\mathrm{EMIM}]\left[\mathrm{AlBr}_{4}\right]_{2}\right\}$-优化构型见图1(e), 具体结构 参数列于表 4 (对阴离子部分相同原子进行编号时按 照从左至右自小而大的方式), 优化结果基本能够反 映出晶体结构中离子间的作用模式. 相对于前面的 离子簇模型, 此模型的优化构型参数偏离实验数据 较大, 最明显的偏离是两个阴离子的中心A1所成直 线与咪唑环平面之间的夹角(锐角)比晶体结构中的 相应数值小, 因而表 4 中二面角及键角数据与实验

表3 [EMIM] $\left[\mathrm{BF}_{4}\right]$ 红外光谱实验及理论计算值

Table 3 Experimental and calculated IR spectrum data of [EMIM][BF

\begin{tabular}{clll}
\hline Experimental IR spectrum $^{11}$ & $\multicolumn{1}{c}{[\mathrm{EMIM}]\left[\mathrm{BF}_{4}\right]_{3}}^{2-}$ & $\multicolumn{1}{c}{[\mathrm{EMIM}]_{2}\left[\mathrm{BF}_{4}\right]_{4}}^{2-}$ & Assignment \\
\hline 3166 & $3247(v \mathrm{C} 4-\mathrm{H}, \mathrm{C} 5-\mathrm{H})$ & 3277 & $v \mathrm{C} 5-\mathrm{H}$ \\
3125 & 3231 & 3240 & $v \mathrm{C} 2-\mathrm{H}$ \\
& $3230(v \mathrm{C} 4-\mathrm{H}, \mathrm{C} 5-\mathrm{H})$ & 3228 & $v \mathrm{C} 4-\mathrm{H}$ \\
2989 & 3162 & $3162(2967)$ & $v_{\text {as }} \mathrm{CH}_{3}(\mathrm{Me})$ \\
$\sim 2970$ & 3140 & $3161(2966)$ & $v_{\text {as }} \mathrm{CH}_{2}(\mathrm{Et})$ \\
2952 & 3147 & $3153(2959)$ & $v_{\text {as }} \mathrm{CH}_{3}(\mathrm{Me})$ \\
$\sim 2917$ & 3117 & $3100-3122(2909-2930)$ & $v_{\text {as }} \mathrm{CH}_{3}(\mathrm{Et})$ \\
& 3101 & & $v_{\text {as }} \mathrm{CH}_{2}(\mathrm{Et})$ \\
2892 & 3079 & $3094(2903)$ & $v_{\text {as }} \mathrm{CH}_{3}(\mathrm{Me})$ \\
& 3067 & $3070(2881)$ & $v_{\text {as }} \mathrm{CH}_{3}(\mathrm{Et})$ \\
1619 & 3033 & $3033(2846)$ & $v_{\text {as }} \mathrm{C}_{2}-\mathrm{N} 3-\mathrm{C} 4-\mathrm{C} 5$ \\
& 1606 & 1611 & $v_{\text {as }} \mathrm{N} 1_{-\mathrm{C} 2}-\mathrm{N} 3$ \\
\hline
\end{tabular}


表4 溴铝酸盐晶体结构及 $\left\{[\mathrm{EMIM}]\left[\mathrm{AlBr}_{4}\right]_{2}\right\}^{-},\left\{[\mathrm{EMIM}]\left[\mathrm{AICl}_{4}\right]_{2}\right\}^{-}$优化构型结构参数

Table 4 Structural parameters in [EMIM] $\left[\mathrm{AlBr}_{4}\right]$ crystal and optimized ion clusters of $\left\{[\mathrm{EMIM}]\left[\mathrm{AIBr}_{4}\right]_{2}\right\}^{-}$and $\left\{[\mathrm{EMIM}]\left[\mathrm{AICl}_{4}\right]_{2}\right\}^{-}$

\begin{tabular}{lccc}
\hline & $\left\{[\mathrm{EMIM}]\left[\mathrm{AlBr}_{4}\right]_{2}\right\}^{-}$ & $\left.\left\{[\mathrm{EMIM}]\left[\mathrm{AlBr}_{4}\right]^{\mathrm{a}}\right]\left[\mathrm{AlCl}_{4}\right]_{2}\right\}^{-}$ \\
\hline$A(\mathrm{~A} 11-\mathrm{C} 2-\mathrm{N} 3-\mathrm{N} 1) /\left({ }^{\circ}\right)$ & 127.7 & 63.6 & 118.2 \\
$A(\mathrm{~A} 12-\mathrm{C} 2-\mathrm{N} 1-\mathrm{N} 3) /\left(^{\circ}\right)$ & 46.5 & 77.1 & 49.9 \\
$A(\mathrm{~A} 11-\mathrm{C} 2-\mathrm{Al} 2) /\left(^{\circ}\right)$ & 173.1 & 150.8 & 175.4 \\
$A(\mathrm{~A} 11-\mathrm{H}(\mathrm{C} 2)-\mathrm{C} 2) /\left({ }^{\circ}\right)$ & 125.8 & 67.5 & 115.4 \\
$A(\mathrm{~A} 12-\mathrm{H}(\mathrm{C} 2)-\mathrm{C} 2) /\left({ }^{\circ}\right)$ & 39.2 & 69.0 & 57.7 \\
$L(\mathrm{Al1}-\mathrm{C} 2) / \mathrm{nm}$ & 0.4183 & 0.4866 & 0.3994 \\
$L(\mathrm{Al} 2-\mathrm{C} 2) / \mathrm{nm}$ & 0.5269 & 0.4596 & 0.4984 \\
\hline
\end{tabular}

${ }^{a}$ from Cambridge Structural Database

表5 离子簇模型中离子间相互作用能及实验熔点

Table 5 Ion interaction energy and experimental melting points of ion cluster model

\begin{tabular}{|c|c|c|c|c|}
\hline System & $\Delta E_{1-n}^{\mathrm{a}} /\left(\mathrm{kJ} \cdot \mathrm{mol}^{-1}\right)$ & $\left(\Delta E_{2-n^{\prime}} / 2\right)^{\mathrm{b}} /\left(\mathrm{kJ} \cdot \mathrm{mol}^{-1}\right)$ & $T_{\mathrm{m}}(\exp )^{22} / \mathrm{K}$ & {$\left[T_{\mathrm{m}}(\mathrm{cal})-T_{\mathrm{m}}(\exp )\right] / \mathrm{K}$} \\
\hline$[\mathrm{EMIM}]\left[\mathrm{BF}_{4}\right]$ & $-391.09(-380.25)$ & $-431.83(-421.88)$ & $288^{32}$ & -41 \\
\hline [EMIM][FHF] & $-459.59(-447.96)$ & $-414.87(-404.20)$ & 324 & 9 \\
\hline$[\mathrm{EMIM}] \mathrm{Cl}$ & $-418.84(-409.93)$ & $-478.04(-468.96)$ & 357 & -24 \\
\hline [EMIM]Br & $-390.77(-385.12)$ & $-449.64(-444.18)$ & 347 & -5 \\
\hline [EMIM]I & $-388.98(-369.12)$ & $-442.61(-423.37)$ & 353 & 8 \\
\hline$[\mathrm{EMIM}]\left[\mathrm{Br}_{3}\right]$ & $-402.80(-399.11)$ & $-386.71(-382.88)$ & 322 & 35 \\
\hline [EMIM][IBrI] & $-395.77(-387.14)$ & $-379.52(-371.26)$ & 282 & 6 \\
\hline$[\mathrm{EMIM}]\left[\mathrm{AlCl}_{4}\right]$ & $-386.92(-375.37)$ & $-372.92(-360.95)$ & 282 & 12 \\
\hline
\end{tabular}

Energy information for ion liquid ions obtained using B3LYP/6-311++G $(d, p)(6-311 \mathrm{G}(d, p)$ basis set was used for I) calculations (data in parentheses were energy values after basis set superposition error (BSSE) correction). ${ }^{a} \Delta E_{1-n}$ represents the interaction energy in $\left\{[\text { EMIM]X }\}^{(n-1)-}\right.$ model, $^{\mathrm{b}} \Delta E_{2-n^{\prime}}$ represents the interaction energy in $\left\{[\mathrm{EMIM}]_{2} \mathrm{X}_{n^{\prime}}\right\}^{\left(n^{\prime}-2\right)-}$ model

值偏离较大, 相应的键长偏离实验数据 $0.07 \mathrm{~nm}$. 当 阴离子尺寸变大, 离子堆叠情况更加复杂时, 周围 离子的影响越来越不可忽略. 同时对 $\left\{[\mathrm{EMIM}]_{2}\left[\mathrm{AlX}_{4}\right]_{3}\right\}$ $(\mathrm{X}=\mathrm{Cl}, \mathrm{Br})$ 进行了构型优化, $\left\{[\mathrm{EMIM}]_{2}\left[\mathrm{AlBr}_{4}\right]_{3}\right\}^{-}$的 优化结果参见图1(f).

\section{2 离子间相互作用能与实验熔点之间的关系}

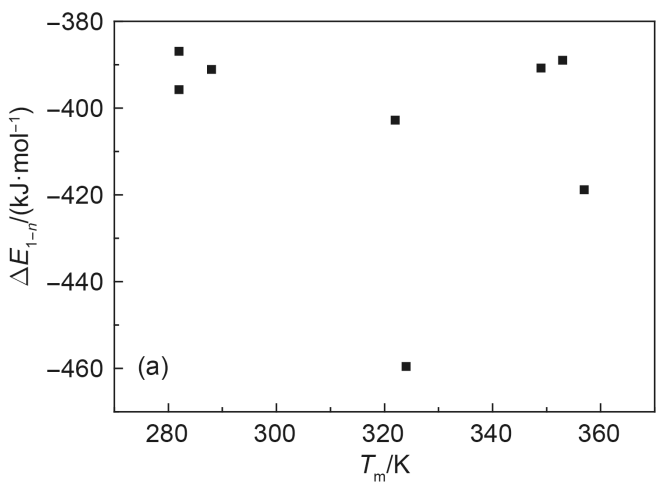

所研究体系的 $\left\{[\mathrm{EMIM}] \mathrm{X}_{n}\right\}^{(n-1)-}(\mathrm{X}=\mathrm{Cl}, \mathrm{Br}, \mathrm{I}$, $\left.\mathrm{BF}_{4}, \mathrm{AlCl}_{4}, \mathrm{Br}_{3}, \mathrm{IBrI}, \mathrm{FHF} ; n=2,3\right)$ 和 $\left\{[\mathrm{EMIM}]_{2} \mathrm{X}_{n^{\prime}}\right\}^{\left(n^{n}-2\right)}$ $\left(n^{\prime}=3,4,5\right)$ 模型的离子间相互作用能列于表 5 中(括 号中数据为经过BSSE校正后的结果). 其中, $\Delta E_{1-n}$ 、 $\Delta E_{2-n}$ 分别代表 $\left\{[\mathrm{EMIM}] \mathrm{X}_{n}\right\}^{(n-1)-}$ 和 $\left\{[\mathrm{EMIM}]_{2} \mathrm{X}_{n^{\prime}}\right\}^{\left(n^{\prime}-2\right)}$ 模 型的阴、阳离子间相互作用能. 从表 5 中数据可以看

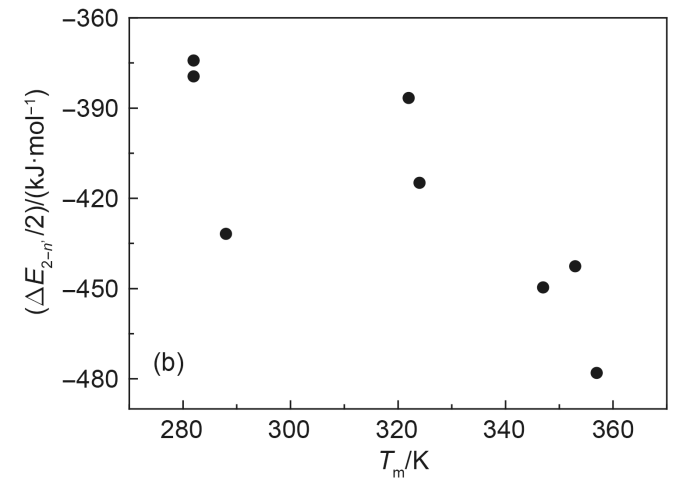

图3 EMIM离子液体离子间相互作用能与实验熔点之间的关系

Fig.3 Relationships between the ion interaction energies and the experimental melting points of EMIM ion liquids (a) $\Delta E_{1-n}-T_{\mathrm{m}}$ data for $\left\{[\mathrm{EMIM}] \mathrm{X}_{n}\right\}^{(n-1)-}$ models $\left(\mathrm{X}=\mathrm{Cl}, \mathrm{Br}, \mathrm{I}_{1} \mathrm{BF}_{4}, \mathrm{AlCl}_{4}, \mathrm{Br}_{3}, \operatorname{IBrI}, \mathrm{FHF} ; n=2,3\right)$;

(b) $\Delta E_{2-n^{\prime}} / 2-T_{\mathrm{m}}$ data for $\left\{[\mathrm{EMIM}]_{2} \mathrm{X}_{n^{\prime}}\right\}^{\left(n^{\prime}-2\right)-} \operatorname{models}\left(n^{\prime}=3,4,5\right)$ 
到除 $[$ EMIM]I体系外BSSE修正数值均较小. 不同体 系的实验熔点 (主要来自文献, ${ }^{22,32}$ 当有不同的熔点被 报道时采用平均值)也列于表 5 中.

图3更直观地反映了实验熔点与各离子簇模型 离子间相互作用能 $\Delta E_{1-n} 、 \Delta E_{2-n} / 2$ 之间的关系. 可以 看到对于阴离子不同的EMIM离子液体，采用 $\left\{[\mathrm{EMIM}]_{2} \mathrm{X}_{n^{\prime}}\right\}^{\left(n^{\prime}-2\right)}$ 模型时实验熔点与阴、阳离子间 相互作用能之间趋向线性关系. 根据 8 个数据点线 性拟合得到方程: $T_{\mathrm{m}}=-125.40-0.92091 \times \Delta E$, 决定 系数 $|R|=0.74$. 采用上述拟合方程预测得到的熔点 值 $T_{\mathrm{m}}$ (cal)与实验值 $T_{\mathrm{m}}(\mathrm{exp})$ 的差值也列于表 5 中. 结果 表明采用能够反映晶体结构特征的多阳离子、多 阴离子的离子簇模型, 阴、阳离子间相互作用能与 实验熔点存在定量关系.

\section{4 结 论}

对1-乙基-3-甲基咪唑卤化物、氟嗍酸盐、三 溴化物和二碘溴酸盐、氯铝酸和溴铝酸盐进行了 离子簇模型研究, 所涉及的EMIM离子液体涵盖了 不同种类、不同尺寸阴离子的体系. 我们首先在 B $3 \mathrm{~L} \mathrm{Y} \mathrm{P/} 6$ - $311++\mathrm{G}(d, p)$ 水平上 (对 $\mathrm{I}$ 使用 6$311 \mathrm{G}(d, p)$ 基组 $)$ 分别对 $\left\{[\mathrm{EMIM}]_{2} \mathrm{X}_{4}\right\}^{2-}(\mathrm{X}=\mathrm{Cl}, \mathrm{Br}, \mathrm{I}$, $\left.\mathrm{BF}_{4}\right) 、\left\{[\mathrm{EMIM}] \mathrm{X}_{2}^{\prime}\right\}^{-} 、\left\{[\mathrm{EMIM}]_{2} \mathrm{X}_{3}^{\prime}\right\}^{-}\left(\mathrm{X}^{\prime}=\mathrm{AlCl}_{4}\right.$, $\left.\mathrm{AlBr}_{4}, \mathrm{Br}_{3}, \mathrm{IBrI}\right) 、\left\{[\mathrm{EMIM}][\mathrm{FHF}]_{3}\right\}^{2-} 、\left\{[\mathrm{EMIM}]_{2}[\mathrm{FHF}]_{5}\right\}^{3-}$ 共14种离子簇进行结构优化, 并对卤化物和氟硼酸 盐进行了振动光谱计算. 结果表明对于所选定的体 系, 多阳离子、多阴离子的离子簇模型能够更好地 反映相应晶体中的结构及阴、阳离子相互作用特 征. 我们同时对阴、阳离子间相互作用能与实验熔 点之间的关系进行了研究, 发现采用 2 个咪唑阳离 子和 $n$ 个阴离子组成的模型时不同类型的EMIM离 子液体晶体实验熔点与阴、阳离子间相互作用能 之间呈现近线性关系. 借助理论计算预言离子液体 性质一直是人们的一个研究目标, 我们在之前离子 液体熔点构效关系研究中发现, 若想获得普适性 强、形式简单、物理意义明确的定量构效关系表 达式需要以合理的结构模型为出发点. 本文的工作 进一步证明了对于离子液体这种特殊的体系, 有可 能利用离子簇模型模拟晶体中的结构特征, 并进一 步通过离子间相互作用能预言实验熔点. 我们希望 通过我们的工作为离子液体的理性化设计提供有 力的理论支持.

\section{References}

(1) Rogers, R. D.; Seddon, K. R. Science 2003, 302, 792. doi: 10.1126/science.1090313

(2) Li, R. X. Green Solvent-the Synthesis and Application of Ionic Liquids; Chemical In dustry Press: Beijing, 2004. [李汝雄. 绿色 溶剂一离子液体的合成与应用. 北京: 化学工业出版社, 2004.]

(3) Zhang, S. J.; Lü, X. M. Ionic Liquids-from Fundamentals to Applications; Scientific Publish Ltd.: Beijing, 2006. [张锁江, 吕 兴梅. 离子液体一从基础研究到工业应用. 北京: 科学出版社, 2006.]

(4) Hallet, J. P.; Welton, T. Chem. Rev. 2011, 111, 3508. doi: $10.1021 / \mathrm{cr} 1003248$

(5) Li, W.; Wu, X. M.; Qi, C. S.; Rong, H.; Gong, L. F. J. Mol. Struct:: Theochem 2010, 942, 19. doi: 10.1016/j.theochem. 2009.11.027

(6) Li, W.; Qi, C. S.; Wu, X. M.; Rong, H.; Gong, L. F. Acta Phys. -Chim. Sin. 2011, 27, 2059. [李 巍, 戚传松, 吴新民, 荣 华, 龚良发. 物理化学学报, 2011, 27, 2059.] doi: 10.3866/PKU. WHXB20110914

(7) Li, W.; Qi, C. S.; Rong, H.; Wu, X. M.; Gong, L. F. Chem. Phys. Lett. 2012, 542, 26. doi: 10.1016/j.cplett.2012.05.072

(8) Wu, Y.; Zhang, T. T. J. Phys. Chem. A 2009, 113, 12995. doi: $10.1021 /$ jp906465h

(9) Mohajeri, A.; Ashrafi, A. J. Phys. Chem. A 2011, 115, 6589. doi: $10.1021 /$ jp1093965

(10) Turner, E. A.; Pye, C. C.; Singer, R. D. J. Phys. Chem. A 2003, 107, 2277. doi: 10.1021/jp021694w

(11) Katsyuba, S. A.; Zvereva, E. E.; Vidis, A.; Dyson, P. J. J. Phys. Chem. A 2007, 111, 352. doi: 10.1021/jp064610i

(12) Xiao, X.; Guo, M.; Pei, Y.; Zheng, Y. Spectrochimica Acta Part A 2011, 78, 1492

(13) Rao, S. S.; Gejji, S. P. Computational and Theoretical Chemistry 2015, 1057, 24. doi: 10.1016/j.comptc.2015.01.012

(14) García, G.; Atilhan, M.; Aparicio, S. Chem. Phys. Lett. 2014, 610-611, 267.

(15) Katritzky, A. R.; Lomaka, A.; Petrukhin, R.; Jain, R.; Karelson, M.; Visser, A. E.; Rogers, R. D. J. Chem. Inf. Comput. Sci. 2002, 42, 71. doi: $10.1021 / \mathrm{ci0} 0100503$

(16) Zhang, S. J.; Sun, N.; He, X. Z.; Lu, X. M.; Zhang, X. P. J. Phys. Chem. Ref. Data 2006, 35, 1475. doi: 10.1063/1.2204959

(17) Varnek, A.; Kireeva, N.; Tetko, I. V.; Baskin, I. I.; Solov'ev, V. P. J. Chem. Inf. Model. 2007, 47, 1111. doi: 10.1021/ci600493x

(18) Ren, Y. Y.; Qin, J.; Liu, H. X.; Yao, X. J.; Liu, M. C. QSAR Comb. Sci. 2009, 28, 1237. doi: 10.1002/qsar.v28:11/12

(19) Kowsari, M. H.; Alavi, S.; Najafi, B.; Gholizadeh, K.; Dehghanpisheh, E.; Ranjbar, F. Phys. Chem. Chem. Phys. 2011, 13, 8826. doi: 10.1039/c0cp02581j

(20) He, L.; Tao, G. H.; Parrish, D. A.; Shreeve, J. M. J. Phys. Chem. B 2009, 113, 15162. doi: 10.1021/jp905079e

(21) Rahman, M. B. A.; Jumbri, K.; Basri, M.; Abdulmalek, E.; Sirat, K.; Salleh, A. B. Molecules 2010, 15, 2388. doi: 10.3390/ molecules 15042388 
(22) The Reaxys Database, http://www.reaxys.com.

(23) Hu, S. Z.; Zhou, Z. H.; Cai, Q. R. Acta Phys. -Chim. Sin. 2003, 19, 1073. [胡盛志, 周朝晖, 蔡启瑞. 物理化学学报, 2003, 19, 1073.] doi: 10.3866/PKU.WHXB20031118

(24) Frisch, M. J.; Trucks, G. W.; Schlegel, H. B.; et al. Gaussian 03, Revision E.01; Gaussian Inc.: Wallingford, CT, 2004.

(25) Morrow, T. I.; Maginn, E. J. J. Phys. Chem. B 2002, 106, 12807. doi: $10.1021 /$ jp0267003

(26) van Duijneveldt, F. B.; van Duijneveldt-van de Rijdt, J. G. C. M.; van Lenthe, J. H. Chem. Rev. 1994, 94, 1873. doi: 10.1021/ cr00031a007

(27) Jansen, H. B.; Ros, P. Chem. Phys. Lett. 1969, 3, 140 doi: 10.1016/0009-2614(69)80118-1
(28) Boys, S. F.; Bernardi, F. Mol. Phys. 1970, 19, 553. doi: 10.1080/00268977000101561

(29) Dymek, C. J.; Grossie, D. A.; Fratini, A. V. J. Mol. Struct. 1989, 213, 25. doi: 10.1016/0022-2860(89)85103-8

(30) Scott, A. P.; Radom, L. J. Phys. Chem. 1996, 100, 16502. doi: 10.1021/jp960976r

(31) Elaiwi, A.; Hitchcock, P. B.; Seddon, K. R.; Srinivasan, N.; Tan, Y. M.; Welton, T.; Zora, J. A. J. Chem. Soc. Dalton Trans. 1995, 3467.

(32) Wang, J.; Yang, X. Z.; Wu, S. D.; Li, G. S. Properties and Applications of Ionic Liquids; China Textile \&Apparel Press: Beijing, 2006. [王 军, 杨许召, 吴诗德, 李刚森. 离子液体的性 能及应用. 北京: 中国纺织出版社, 2006.] 